\title{
Local elastic expansion model for viscous-flow activation energies of glass-forming molecular liquids
}

\author{
Jeppe C. Dyre, Niels Boye Olsen, and Tage Christensen \\ Department of Mathematics and Physics (IMFUFA), Roskilde University, P.O. Box 260, DK-4000 Roskilde, Denmark
}

(Received 27 June 1995; revised manuscript received 15 August 1995)

\begin{abstract}
A model for the viscosity of glass-forming molecular liquids is proposed in which a "flow event" requires a local volume increase. The activation energy for a flow event is identified with the work done in shoving aside the surrounding liquid; this work is proportional to the high-frequency shear modulus, which increases as the temperature decreases. The model is confirmed by experiments on a number of molecular liquids.
\end{abstract}

Glass formation is a universal property of supercooled liquids. ${ }^{1-9}$ For simple liquids rapid cooling is required to avoid crystallization. For most complex liquids supercooling causes no problems; in fact, many complex liquids are difficult to crystallize. The glass transition takes place when the viscosity of the supercooled liquid becomes so large that molecular motion is arrested. The laboratory glass transition is dynamic and not a phase transition, although many workers in the field believe it to be a manifestation of an underlying equilibrium second-order phase transition. For cooling rates of order Kelvin per minute, the glass transition takes place when the viscosity, $\eta$, is around $10^{13}$ poise $(\mathrm{P})$. In the following, the glass transition temperature, $T_{g}$, is defined as the temperature at which $\eta=10^{13} \mathrm{P}$.

The linear shear mechanical properties of a liquid are determined by the shear modulus as function of frequency, $G(\omega)=G^{\prime}(\omega)+i G^{\prime \prime}(\omega)$. At low frequencies $G(\omega)=i \omega \eta$. At high frequencies liquid becomes solidlike and $G(\omega)$ approaches a limiting value, $\lim _{\omega \rightarrow \infty} G(\omega)=G_{\infty}$. In terms of $\eta$ and $G_{\infty}$, the average shear relaxation time, $\tau$, is given ${ }^{3}$ by Maxwell's expression

$$
\tau=\frac{\eta}{G_{\infty}}
$$

For all viscous liquids $\tau$ and $\eta$ depend dramatically on temperature, varying often more than ten decades over a temperature range quite narrow compared to $T_{g}$. $G_{\infty}$ depends much less on temperature, usually increasing less than a factor of 4 upon cooling in the same temperature range. This variation, on the other hand, is considerably larger than that found in simple nonviscous liquids or in crystals and glasses.

Intimately linked to the problem of understanding the glass transition is the problem of the temperature dependence of viscosity: Upon cooling the viscosity increases more than expected from a simple Arrhenius law (exceptions to this are $\mathrm{SiO}_{2}$ and $\mathrm{GeO}_{2}$ ). There is still no consensus regarding what causes the non-Arrhenius temperature dependence of viscosity. The two most important phenomenological models are the free volume model of Grest and Cohen, ${ }^{10}$ and the entropy model of Gibbs and co-workers. ${ }^{11,12}$ In the free volume model, the viscosity is controlled by the volume available for molecular rearrangements, which decreases with decreasing temperature. In the entropy model, the increase in viscosity upon cooling is caused by the decrease in the configurational entropy. ${ }^{13}$ In the less viscous regime, the non-Arrhenius viscosity is explained by mode-coupling theories. ${ }^{14,15}$

It is generally believed that flow in viscous liquids proceeds via sudden flow events involving several molecules. ${ }^{3,10,12,16-21}$ In terms of the free energy barrier to be overcome, $\Delta F(T)$, the temperature dependence of the viscosity is given by

$$
\eta=\eta_{0} \exp \left[\frac{\Delta F(T)}{k_{B} T}\right]
$$

According to Eq. (2) the non-Arrhenius temperature dependence of the viscosity arises because $\Delta F(T)$ increases as the temperature decreases. The problem is to explain why.

In molecular liquids van der Waals forces (and possibly hydrogen bonding) are present between the molecules. The starting point for the present model is the fact that the repulsions between the molecules are strong, while the attractions are only weak. Many properties of simple liquids derive from this fact. ${ }^{22}$ In a viscous liquid a flow event involves a significant rearrangement of a number of densely packed molecules. If the flow event takes place at a constant volume, the molecules are forced into close contact. Because of the strong repulsions between the molecules, this is energetically very costly. Alternatively, the molecules may shove aside the surrounding liquid to increase the volume available for rearranging. This must be less costly than rearranging at a constant volume; consequently, the new model is based on "shoving" flow events.

The barrier height has two contributions, one from shoving aside the surrounding liquid and one from separating the flow event molecules. In a harmonic solid, as is easy to show, the two contributions are of the same order of magnitude, but the relative distance changes between the flow event molecules are too large for using the harmonic approximation. Since the attractive forces are only weak, the energy cost for separating the flow event molecules is considerably lower than estimated from the harmonic approximation. We ignore this contribution and identify $\Delta F(T)$ with the shoving work done on the surrounding liquid.

Like any thermally activated transition, a flow event is a rapid process. During the shoving the surrounding liquid behaves like a solid, and the shoving work depends linearly on the infinite-frequency bulk and shear moduli, $K_{\infty}$ and $G_{\infty}$. To 

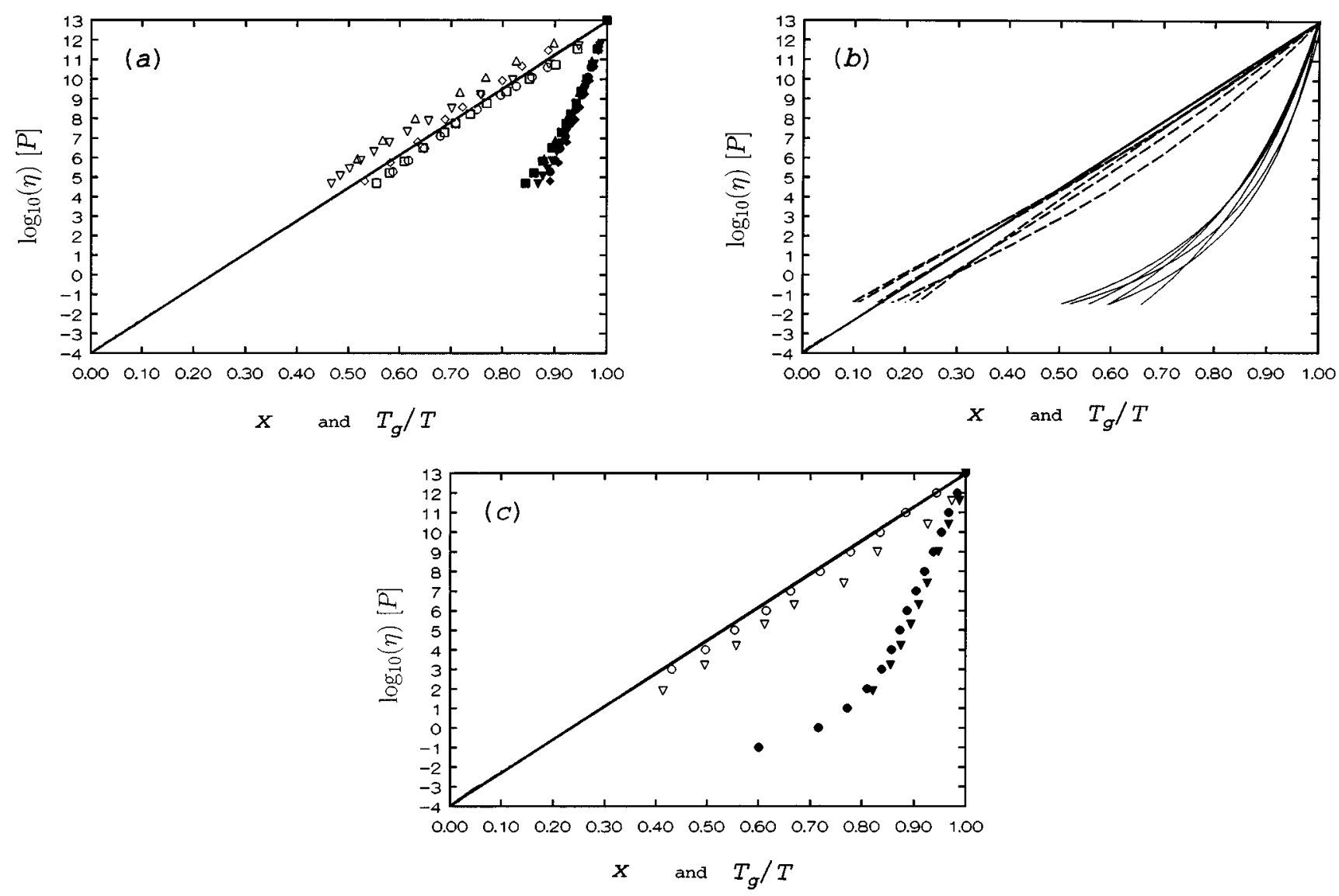

FIG. 1. Logarithm of the viscosity in Poise as function of $T_{g} / T$ and as function of the variable $x \propto G_{\infty}(T) / T$ normalized to one at $T=T_{g}$ : $x=\left[G_{\infty}(T) T_{g}\right] /\left[G_{\infty}\left(T_{g}\right) T\right]$. According to the model the logarithm of the viscosity depends linearly on $x$ [Eqs. (2) and (3)]. In each subfigure the diagonal line joins the point $x=1$ and $\log _{10}(\eta)=13$ with the point $x=0$ and $\log _{10}(\eta)=-4$. The former point defines the glass transition and the latter corresponds to the viscosity prefactor of Eq. (2), $\eta_{0}$, equal to $10^{-4} \mathrm{P}$ (a typical prefactor, corresponding to the shear relaxation time close to an average period of vibration) [Ref. 24]. The subfigures give data where $G_{\infty}(T)$ was obtained from extrapolations of measurements in different frequency ranges (a) $\mathrm{mHz}-\mathrm{kHz}$; (b) $\mathrm{MHz}$; (c) GHz. (a) shows our data for five molecular liquids. These liquids all accurately obey the time-temperature superposition principle for the shear modulus (except for a $\beta$ component with a magnitude less than $10 \%)$. Consequently, the Kramers-Kronig sum rule $G_{\infty}=(2 / \pi) \int_{-\infty}^{\infty} G^{\prime \prime}(\omega) d \ln \omega$ implies that $G_{\infty}$ is proportional to the maximum loss, $G_{\max }^{\prime \prime}$, which makes it possible to evaluate the variable $x$ directly from data without any analytical extrapolation: $x$ $=\left[G_{\max }^{\prime \prime}(T) T_{g}\right] /\left[G_{\max }^{\prime \prime}\left(T_{g}\right) T\right]$. In (a) the full symbols give the viscosity as function of $T_{g} / T$ and the open symbols give the viscosity as function of $x$. The figure shows data for 4-methylpentan-2-ol $(\triangle)$, dioctyl phthalate $(\square)$, phenyl salicylate (salol) $(\bigcirc)$, dibutyl phthalate $(\nabla)$, and the silicone oil MS704 $(\diamond)$. (b) gives the data of Barlow et al. (Ref. 28), where $G_{\infty}$ was obtained from extrapolations of ultrasonic measurements (Ref. 2). The full curves give the viscosities as function of $T_{g} / T$ and the dashed curves give the viscosities as function of $x$ for the following six molecular liquids: isopropyl benzene, $n$-propyl benzene, sec.-butyl benzene, di(isobutyl)phthalate, di(n-butyl)phthalate, di(2ethyl hexyl)phthalate. (c) gives data where $G_{\infty}$ was obtained from depolarized Brillouin scattering. As in (a) the full symbols give the viscosity as function of $T_{g} / T$ and the open symbols give the viscosity as function of $x$. The figure shows data for 5-phenyl-4-ether ( $\bigcirc$ ) (Ref. 42) and for $\alpha$-phenyl-o-cresol ( $\nabla$ ) (Refs. 43 and 44).

be specific, we assume that shoving increases the volume of the flow event molecules from a sphere to a larger sphere. According to elasticity theory ${ }^{23}$ this induces a radial displacement in the surroundings, $u_{r}$, varying as $u_{r} \propto r^{-2}$. This is a pure shear displacement $(\nabla \cdot \mathbf{u}=0$ as for the Coulomb field) and therefore the shoving work is independent of $K_{\infty}$ and proportional to $G_{\infty}$. The constant of proportionality will be referred to as the characteristic volume, $V_{c}$. For simplicity $V_{c}$ is assumed to be temperature independent, and thus

$$
\Delta F(T)=G_{\infty}(T) V_{c}
$$

The characteristic volume is not equal to the volume change during shoving, the activation volume, $\Delta V$. For small acti- vation volume it is easy to show that $V_{c}$ is given by (where $V$ is the volume before the shoving)

$$
V_{c}=\frac{2}{3} \frac{(\Delta V)^{2}}{V} .
$$

Since $G_{\infty}(T)$ increases upon cooling, the model predicts a non-Arrhenius viscosity with an activation energy that increases as the glass transition is approached. This is what is observed in experiments. On the other hand, the model is inconsistent with the popular Vogel-Fulcher-Tammann expression, ${ }^{6,7}$ where the viscosity diverges at a finite temperature (unless one accepts that $G_{\infty}$ may become infinite which seems unphysical). 
According to the new model the logarithm of the viscosity depends linearly on $G_{\infty}(T) / T$. Figure 1 compares this prediction to experiments in Angell's fragility plots ${ }^{24}$ where the logarithm of the viscosity (in poise) is plotted as function of $x \propto 1 / T$, and as function of $x \propto G_{\infty}(T) / T$ (both $x$ coordinates are normalized to one at $T=T_{g}$ ). We have measured $G(\omega)$ for five molecular liquids using the piezoelectric shear modulus gauge transducer (PSG) consisting of three piezoceramic discs, ${ }^{25}$ a device based on principles similar to those of the bulk modulus transducer. ${ }^{26}$ With recent improvements ${ }^{27}$ the PSG is now able to provide shear modulus data in the frequency range $1 \mathrm{mHz}-50 \mathrm{kHz}$. Figure 1(a) shows our results for the viscosity as function of $T_{g} / T$ (full symbols) and as function of $x$ (open symbols). The line connects the point $x=1$ and $\log _{10} \eta=10^{13}$ (defining the glass transition) with the point $x=0$ and $\log _{10} \eta=10^{-4}$ [a typical viscosity prefactor, corresponding to the average shear relaxation time of Eq. (1) close to an average period of vibration $\left.^{24}\right]$. The results of Fig. 1(a), while favorable for the model, ignore possible additional high-frequency relaxations outside the frequency range covered by the PSG. To investigate whether high-frequency methods for measuring $G_{\infty}$ confirm our findings, we plot data taken from the literature in Figs. 1(b) and 1(c). Figure 1(b) presents the data of Barlow et $a l .{ }^{28}$ for $G_{\infty}$ obtained from ultrasonic measurements working in the $\mathrm{MHz}$ range. Figure $1(\mathrm{c})$ presents data where $G_{\infty}$ was obtained from depolarized Brillouin scattering, a technique that operates in the $\mathrm{GHz}$ range.

Given the uncertainties in evaluating $G_{\infty}$ and the crudeness of the new model, Fig. 1 shows a satisfactory agreement between the model and experiment for molecular liquids. We have also compared the model to Brillouin data for two nonmolecular liquids, ${ }^{29} \mathrm{~B}_{2} \mathrm{O}_{3}$ and $\mathrm{Ca}_{0.4} \mathrm{~K}_{0.6}\left(\mathrm{NO}_{3}\right)_{1.4}(\mathrm{CKN})$. For $\mathrm{B}_{2} \mathrm{O}_{3}, G_{\infty}$ is too dependent on temperature to account for the rather weak non-Arrhenius viscosity. However, viscous flow of $\mathrm{B}_{2} \mathrm{O}_{3}$ involves the breaking of covalent bonds, which goes beyond the present model. For CKN the model works well for the temperature dependence of the conductivity relaxation time [which decouples from the viscosity close to $T_{g}$ (Ref. 30)], but the model is not able to fully explain the dramatic non-Arrhenius viscosity.

We now briefly discuss related work. The idea that volume is needed for a flow event to take place is old; this is the basic idea behind the free volume model. ${ }^{10}$ Here, however, the problematic concept of a "free" volume is extraneous. The present picture is more closely related to that of Brawer, who assumed that the transition state for a flow event is a low-density state with room for the molecules to rearrange. ${ }^{3}$ In his approach, the activation energy was taken to be a function of the energy of the flow event molecules, and the shoving work is ignored. ${ }^{3,19}$ The model proposed here is very similar to that used by Fourkas, Benigno, and Berg ${ }^{31,32}$ for explaining the hole-burning spectra and the time-dependent Stokes' shift of a nonpolar solute molecule in a glassforming solvent. These authors argued that an electronic excitation increases the effective size of the solute molecule, and showed that the viscoelastic response of the surrounding solvent changes the transition energy and causes a timedependent Stokes' shift. Equation (3) appeared in 1968 in two papers by $\mathrm{Nemilov}^{33,34}$ in a version where $V_{c}=V$. Nemilov used Eq. (3) for calculating the rate of flow of optical silicate glasses. He justified Eq. (3) by substituting Eyring's expression for $\eta$ (Ref. 35) and Dushman's expression for $\tau$ (Ref. 36) into Eq. (1). Buchenau and Zorn ${ }^{37,38}$ found empirically that the viscosity of selenium follows the expression $\eta=\eta_{0} \exp \left[u_{0}^{2} /\left\langle u^{2}\right\rangle_{\text {loc }}\right]$, where $\left\langle u^{2}\right\rangle_{\text {loc }}$ is the atomic meansquare displacement for the vibrational motions in the liquid minus the same quantity for the crystal (at the same temperature). This result is related to, but not identical to, that of the present model: If the high-frequency bulk and shear moduli are identical (denoted by $M_{\infty}$ ) and the interatomic harmonic potential is denoted by $(1 / 2) m \omega^{2} u^{2}$, the equipartition theorem implies that $\omega^{2}\left\langle u^{2}\right\rangle \propto T$. The sound velocity is proportional to $\omega$ and to $M_{\infty}^{1 / 2}$. Combining these facts: $M_{\infty}\left\langle u^{2}\right\rangle \propto T$ and thus Eqs. (2) and (3) imply $\eta \propto \exp \left[C /\left\langle u^{2}\right\rangle\right]$. If the difference between $\left\langle u^{2}\right\rangle_{\text {loc }}$ and $\left\langle u^{2}\right\rangle$ is ignored, this is the result of Buchenau and Zorn. As shown by Hall and Wolynes ${ }^{39}$ the relation $\eta \propto \exp \left[C /\left\langle u^{2}\right\rangle\right]$ may be derived by assuming a fixed distance between two minima for harmonic potentials: The energy difference between one minimum and the intersection of the two harmonic potentials varies as $\omega^{2}$, which is proportional to $T /\left\langle u^{2}\right\rangle$. A convincing example of this relation between an activation energy and a phonon frequency was given by Köhler and Herzig, ${ }^{40}$ who were able to explain a number of anomalies for self-diffusion in bcc metals. In their model, the fact that the activation energy for self-diffusion in bcc metals decreases as the temperature decreases, is due to softening of the 111 phonon. Finally, Miles, Le, and Kivelson in a study of the pressure dependence of the sound velocity in triphenylphosphite found that the transverse sound velocity is solely a function of the viscosity. ${ }^{41}$ While their measurements were performed in the less viscous regime, this result is what is expected from the present model (although here viscosity is a function of the transverse sound velocity instead of vice versa), ignoring an insignificant factor $T$.

This work was supported by the Danish Natural Science Research Council.

\footnotetext{
${ }^{1}$ W. Kauzmann, Chem. Rev. 43, 219 (1948).

${ }^{2}$ G. Harrison, The Dynamic Properties of Supercooled Liquids (Academic, New York, 1976).

${ }^{3} \mathrm{~S}$. Brawer, Relaxation in Viscous Liquids and Glasses (American Ceramic Society, Columbus, Ohio, 1985).

${ }^{4}$ J. Jäckle, Rep. Progr. Phys. 49, 171 (1986).

${ }^{5}$ C. A. Angell, J. Phys. Chem. Solids 49, 863 (1988).

${ }^{6}$ G. W. Scherer, J. Non-Cryst. Solids 123, 75 (1990).
}

\author{
${ }^{7}$ C. A. Angell, J. Non-Cryst. Solids 131-133, 13 (1991). \\ ${ }^{8}$ A. Hunt, J. Non-Cryst. Solids 160, 183 (1993). \\ ${ }^{9}$ I. M. Hodge, J. Non-Cryst. Solids 169, 211 (1994). \\ ${ }^{10}$ G. S. Grest and M. H. Cohen, Adv. Chem. Phys. 48, 455 (1981). \\ ${ }^{11}$ J. H. Gibbs and E. A. DiMarzio, J. Chem. Phys. 28, 373 (1958). \\ ${ }^{12}$ G. Adam and J. H. Gibbs, J. Chem. Phys. 43, 139 (1965). \\ ${ }^{13}$ E. A. DiMarzio, Ann. N.Y. Acad. Sci. 371, 1 (1981). \\ ${ }^{14}$ S. P. Das and G. F. Mazenko, Phys. Rev. A 34, 2265 (1986).
}


${ }^{15}$ W. Götze and L. Sjögren, Rep. Progr. Phys. 55, 241 (1992).

${ }^{16}$ M. Goldstein, J. Chem. Phys. 51, 3728 (1969).

${ }^{17}$ G. Williams, in Dielectric and Related Molecular Processes, Specialist Periodical Report, Vol. 2, edited by M. Davies (Chemical Society, London, 1975), p. 151.

${ }^{18}$ E. Donth, Glasübergang (Akademie-Verlag, Berlin, 1981).

${ }^{19}$ J. C. Dyre, Phys. Rev. Lett. 58, 792 (1987).

${ }^{20}$ F. H. Stillinger, J. Chem. Phys. 89, 6461 (1988).

${ }^{21}$ R. V. Chamberlin, Phys. Rev. B 48, 15638 (1993).

${ }^{22}$ D. Chandler, J. D. Weeks, and H. C. Andersen, Science 220, 787 (1983).

${ }^{23}$ L. D. Landau and E. M. Lifshitz, Theory of Elasticity, 2nd ed. (Pergamon, London, 1970).

${ }^{24}$ C. A. Angell, in Relaxations in Complex Systems, edited by K. L. Ngai and G. B. Wright (U.S. G.P.O., Washington, DC, 1985), p. 3.

${ }^{25}$ T. Christensen and N. B. Olsen, J. Non-Cryst. Solids 172-174, 357 and 362 (1994).

${ }^{26}$ T. Christensen and N. B. Olsen, Phys. Rev. B 49, 15396 (1994).

${ }^{27}$ T. Christensen and N. B. Olsen, Rev. Sci. Instrum. 66, 5019 (1995)

${ }^{28}$ A. J. Barlow, J. Lamb, A. J. Matheson, P. R. K. L. Padmini, and J. Richter, Proc. R. Soc. London Ser. A 298, 467 (1967).

${ }^{29}$ M. Grimsditch, R. Bhadra, and L. M. Torell, Phys. Rev. Lett. 62, 2616 (1989).
${ }^{30}$ F. S. Howell, R. A. Bose, P. B. Macedo, and C. T. Moynihan, J. Phys. Chem. 78, 639 (1974).

${ }^{31}$ J. T. Fourkas, A. Benigno, and M. Berg, J. Chem. Phys. 99, 8552 (1993).

${ }^{32}$ J. T. Fourkas, A. Benigno, and M. Berg, J. Non-Cryst. Solids 172-174, 234 (1994).

${ }^{33}$ S. V. Nemilov, Dokl. Akad. Nauk SSSR 181, 1427 (1968).

${ }^{34}$ S. V. Nemilov, Zh. Fiz. Khim. 42, 1391 (1968) [Russ. J. Phys. Chem. 42, 726 (1968)].

${ }^{35}$ H. Eyring, J. Chem. Phys. 4, 283 (1936).

${ }^{36}$ S. Dushman, J. Franklin Inst. 184, 515 (1920).

${ }^{37}$ U. Buchenau and R. Zorn, Europhys. Lett. 18, 523 (1992).

${ }^{38}$ U. Buchenau, Philos. Mag. B 65, 303 (1992).

${ }^{39}$ R. W. Hall and P. G. Wolynes, J. Chem. Phys. 86, 2943 (1987).

${ }^{40}$ U. Köhler and C. Herzig, Philos. Mag. A 58, 769 (1988).

${ }^{41}$ D. G. Miles, Jr., N. Le, and D. Kivelson, J. Chem. Phys. 90, 5327 (1989).

${ }^{42}$ C. T. Moynihan, P. B. Macedo, C. J. Montrose, P. K. Gupta, M. A. DeBolt, J. F. Dill, B. E. Dom, P. W. Drake, A. J. Easteal, P. B. Elterman, R. P. Moeller, H. Sasabe, and J. A. Wilder, Ann. N.Y. Acad. Sci. 279, 15 (1976).

${ }^{43}$ C. H. Wang, R.-J. Ma, and Q.-L. Liu, J. Chem. Phys. 80, 617 (1984).

${ }^{44}$ W. T. Laughlin and D. R. Uhlmann, J. Phys. Chem. 76, 2317 (1972). 